\title{
¿El descortezamiento de un árbol medicinal impacta en su estructura poblacional-espacial? El caso de Hintonia latiflora en México
}

\author{
Does commercial debarking of a medicinal tree impact its population- \\ spatial structure? The case of Hintonia latiflora in Mexico \\ Vicente Florencio Vásquez-Cortez', Leonardo Beltrán-Rodríguez $2^{*}$, Gregorio Ángeles-Pérez', \\ Angélica Romero-Manzanares ${ }^{3}$, Edmundo Garcia-Moya ${ }^{3}$, Mario Luna-Cavazos ${ }^{3}$, Javier Caballero ${ }^{2 \dagger}$, \\ José Blancas ${ }^{4}$, Andrea Martínez-Ballesté ${ }^{2}$ y Francisco Montoya-Reyes ${ }^{5}$
}

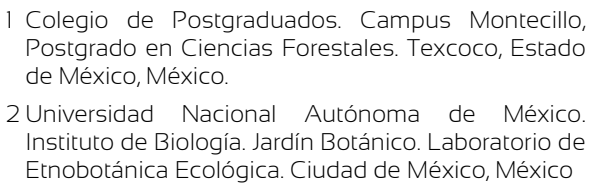
Postgrado en Ciencias Forestales. Texcoco, Estado de México, México.

2 Universidad Nacional Autónoma de México. Instituto de Biología. Jardín Botánico. Laboratorio de Etnobotánica Ecológica. Ciudad de México, México

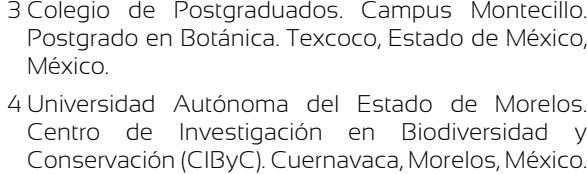

4 Universidad Autónoma del Estado de Morelos. Centro de Investigación en Biodiversidad y Conservación (CIByC). Cuernavaca, Morelos, México.

\author{
5 Instituto Nacional de Investigaciones Forestales, \\ Agrícolas y Pecuarias. Campo Experimental \\ Chetumal. Bacalar, Quintana Roo, México. \\ * Autor de correspondencia. \\ leonbeltranrodríguez@gmail.com
}

\section{RESUMEN}

Se documenta la estructura poblacional y el patrón de distribución y correlación espacial entre etapas de desarrollo de Hintonia latiflora, especie amenazada en México por la cosecha comercial de su corteza. Se establecieron seis unidades de muestreo (tres cosechadas y tres no cosechadas) de $20 \mathrm{~m} \times 100 \mathrm{~m}$ para el censo de todas las plantas y se categorizaron en tres etapas de desarrollo con base en la altura total y diámetro basal; todos los individuos fueron ubicados por sus coordenadas cartesianas. La estructura poblacional se analizó mediante un histograma de densidad, mientras que el sesgo y la curtosis se usaron para determinar el efecto del aprovechamiento sobre las categorías de tamaño. Los patrones espaciales univariantes y bivariantes se determinaron usando la función $L_{1}(t)$ y $L_{12}(t)$ de Ripley, respectivamente, y la significancia estadística fue a 99\% mediante simulaciones Monte Carlo. La densidad entre sitios cosechados $v$ s. no cosechados fue estadísticamente diferente $(\mathrm{p}<0.013)$, pero la estructura poblacional fue semejante, con un patrón de distribución asimétrico positivo $\left(S^{3}=0.947\right.$ y $\left.S^{3}=1.063\right)$ y leptocúrtico $\left(S^{4}=2.006\right.$ y $\left.S^{4}=2.400\right)$. El patrón de distribución univariante en todos los casos fue agregado $(L(t)>0)$, mientras que la correlación espacial en poblaciones sujetas a descortezamiento reflejó una combinación entre independencia y atracción espacial a diferentes distancias. Esta información aporta bases para el manejo sostenible de $H$. latiflora y representa el primer estudio en México que analiza el impacto del descortezamiento de un recurso medicinal sobre su estructura poblacional-espacial.

PALABRAS CLAVE: copalchi, cosecha de corteza, ecología espacial, $K(t)$ de Ripley, quina, recursos forestales no maderables.

\section{ABSTRACT}

The population structure and the patterns of spatial distribution and correlation among developmental stages of Hintonia latiflora, a species threatened in Mexico by the commercial harvest of its bark, are documented. Six plots (three in harvested sites and three in not harvested ones) of $20 \mathrm{~m} \times 100 \mathrm{~m}$ were established for the census of all plants and they were categorized into three stages of development based on the total height and basal diameter; all individuals were located by their cartesian coordinates. The population structure was analyzed by a density histogram; whereas bias and kurtosis were used to determine the harvest effect on size categories. Univariate and bivariate spatial patterns were determined using Ripley's $L(t)$ and $L_{12}(t)$ functions, respectively, and the statistical significance was $99 \%$ using Monte Carlo simulations. Density between harvested sites $v$ s. not harvested was statistically different $(\mathrm{p}<0.013)$, but the population structure was similar, with a positive asymmetric distribution pattern $\left(S^{3}=0.947\right.$ and $\left.S^{3}=1.063\right)$ and leptokurtic $\left(S^{4}=2.006\right.$ and $\left.S^{4}=2.400\right)$. The univariate distribution pattern in all cases was aggregated $(L(t)>0)$, whereas the spatial correlation in populations subject to debarking reflected a combination between independence and spatial attraction at different distances. This information provides a basis for the sustainable management of $H$. latiflora and represents the first study in Mexico that analyzes the debarking of a medicinal resource on its population-spatial structure.

KEYWORDS: copalchi, bark harvest, spatial ecology, Ripley's K(t), quina, non-timber forest resources. 


\section{INTRODUCCIÓN}

El análisis espacial facilita determinar la respuesta de los organismos frente a escenarios naturales y recursos ambientales finitos y heterogéneos en el espacio, lo que condiciona en gran medida el funcionamiento y desarrollo estructural de estos en tiempo evolutivo (McIntire y Fajardo, 2009; Soberón y Miller, 2009). Debido a ello, la distribución espacial es un proceso ecológico altamente importante en el estudio de la estructura y dinámica poblacional de las especies arbóreas, dado que influye en las tasas vitales que permiten estimar la reproducción, crecimiento y mortalidad de una población (Badii, Guillen, Cerna y Landeros, 2011).

Algunos autores han señalado que en los bosques tropicales existe una mayor complejidad estructural y una elevada riqueza de especies (Koleff, Urquiza-Haas y Contreras, 2012; Zakaria, Rajpar, Ozdemir y Rosli, 2016). La distribución espacial de estas suele presentar cierta tendencia a la regularidad en escalas espaciales de paisaje (Ledo, Condés y Montes, 2012); sin embargo, al reducir la escala de análisis se adquiere una fuerte tendencia a formar agregados, particularmente de especies en estadios iniciales durante la sucesión y de hábitats fragmentados (Lawes et al., 2008; Picard, Bar-Hen, Mortier y Chadoeuf, 2009). Los patrones de distribución espacial de especies maderables y no maderables se encuentran estrechamente relacionados con diversos procesos auto y sinecológicos de estos bosques (Rayburn, Schiffers y Schupp, 2011; van Lent et al., 2014), así como en respuesta al efecto antropógeno sobre el recurso (Martínez-Ballesté y Martorell, 2015; Jansen, Anten, Bongers, Martínez-Ramos y Zuidema, 2018).

En los bosques tropicales caducifolios del Neotrópico, el efecto de la fragmentación del hábitat ha impactado negativamente en la biología reproductiva y los síndromes de polinización de diversas especies arbóreas, lo que se considera podría afectar procesos como la dispersión de semillas y, en consecuencia, los patrones de distribución espacial (Quesada et al., 2011). A su vez, el aprovechamiento de especies arbóreas también provoca cambios en la distribución de las especies. En México, van Lent,
Hernández-Barrios, Anten y Martínez-Ramos (2014) evaluaron que la defoliación con propósito comercial de Chamaedorea ernesti-angustii afecta la producción de frutos y la habilidad para dispersar las semillas. Otros estudios han determinado que la cosecha de flores en el árbol medicinal Tilia mexicana impacta críticamente en la distribución espacial de las poblaciones (Pavón-Hernández y Rico-Gray, 2004), debido a que en sitios donde las actividades de recolección son comunes el patrón espacial es azaroso, mientras que en sitios donde no se cosecha por la lejanía y el difícil acceso, el patrón tiende a ser agregado.

A la fecha, pese a la importancia del bosque tropical caducifolio como fuente de abastecimiento de diversos recursos forestales no maderables (RFNM) en México (Blancas, Caballero y Beltrán-Rodríguez, 2017), poco es lo que se conoce sobre la respuesta ecológica de las plantas cosechadas y los efectos sobre su estructura poblacional y distribución espacial (Hernández-Apolinar, Valverde y Purata, 2006; Arellanes-Cancino, Romero-Sosa, Vega, Maza-Villalobos y Casas, 2018), y menos aun en aquellas especies arbóreas en riesgo ecológico por la demanda intensiva de su corteza (Beltrán-Rodríguez et al., 2015; Beltrán-Rodríguez, Manzo-Ramos, Maldonado-Almanza, Martínez-Ballesté y Blancas, 2017a; Beltrán-Rodríguez, 2018a; Beltrán-Rodríguez et al., 2018b).

Hintonia latiflora (Rubiaceae) "quina amarilla" o "copalchi”" es uno de los RFNM más vulnerables en México por el descortezamiento comercial (Beltrán-Rodríguez, Romero-Manzanares, Luna-Cavazos y García-Moya, 2017b). Algunos estudios han demostrado que la cosecha de corteza en los árboles de quina modifica el modelo arquitectónico (mesótono vs basítono), patrón morfométrico (diámetro basal, grosor corteza y altura de la planta) y en el caso de la población, reduce la densidad (Beltrán-Rodríguez et al., 2015; Beltrán-Rodríguez et al., 2017b); pero se desconoce su efecto en cuanto a los cambios de la estructura poblacional cuando las plantas están sujetas o no a descortezamiento parcial o total, así como los efectos que estas prácticas pueden tener en la organización espacial de los individuos de estas poblaciones. 


\section{OBJETIVOS}

Analizar el impacto del aprovechamiento de corteza en $H$. latiflora sobre la estructura poblacional y el patrón de distribución y correlación espacial entre etapas de desarrollo. Se plantea que el descortezamiento insostenible de los árboles de $H$. latiflora provoca la muerte de los individuos (Beltrán-Rodríguez et al., 2015), lo que produce claros en el plano espacial que modifican las condiciones del sitio y las interacciones intraespecíficas, por lo que como respuesta ecológica se encontrarán diferencias estadísticas en la estructura poblacional y espacial en sitios cosechados vs. no cosechados. La estructura poblacional en las áreas no cosechadas mostrará asimetría negativa y las plantas de esta especie en los sitios cosechados tenderán hacia la aleatoriedad, mientras que las diferentes etapas de desarrollo (plántulas, juveniles y adultos) mostrarán independencia espacial para evitar competencia por espacio y recursos.

\section{MATERIALES Y MÉTODOS}

\section{Especie de estudio}

Hintonia latiflora es una especie leñosa, iterópara y hermafrodita, protándrica y hercógama, polinizada por lepidópteros nocturnos y quirópteros, con dispersión anemócora y barócora (Beltrán-Rodríguez et al., 2015). Los individuos son reproductivos a partir de $8 \mathrm{~cm}$ de diámetro basal y, en estos, sus dimensiones alométricas van de $15 \mathrm{~cm}$ a $40 \mathrm{~cm}$ en diámetro basal, $6 \mathrm{~m}$ a $8 \mathrm{~m}$ de altura y ancho de copa de $3.5 \mathrm{~m}$ a $6.5 \mathrm{~m}$, con una producción promedio de corteza en peso seco que oscila entre $500 \mathrm{~g}$ y $4 \mathrm{~kg}$ por individuo (Beltrán-Rodríguez et al., 2017b). Esta especie es endémica del bosque tropical caducifolio de México y Centroamérica (Lorence y Taylor, 2011), y presenta poblaciones aisladas con baja densidad de individuos reproductivos (5 ind. ha ${ }^{-1}$ ) (Beltrán-Rodríguez et al., 2015). La corteza de $H$. latiflora es un RFNM que se recolecta mediante machete a nivel del tallo y ramas, ya sea de plantas en pie o derribadas, prácticas que generalmente afectan la corteza interna y causan la muerte del individuo (BeltránRodríguez et al., 2015). El aprovechamiento se realiza con fines comerciales y sin regulación oficial en México desde 1970 (Hersch-Martínez y Fierro, 2001), distribuyéndose nacional e internacionalmente como antidiabético y febrífugo (Mata, Navarrete, Cristians, Hersch y Bye, 2009); demanda que ha ocasionado extinciones locales y regionales de esta especie, situando a sus poblaciones silvestres en estatus de vulnerabilidad ecológica (Beltrán-Rodríguez et al., 2017a).

\section{Área de estudio}

La investigación se llevó a cabo en la región conocida como Alto Balsas, en el estado de Guerrero, México (17 ${ }^{\circ} 49^{\prime}$ $20^{\circ} 00^{\prime} \mathrm{N}$ y $99^{\circ} 03^{\prime}-99^{\circ} 13^{\prime} \mathrm{W}$ ); área de distribución natural y de cosecha comercial de corteza de H. latiflora. El clima, de acuerdo con García (2004), es $\mathrm{BS}_{1}\left(\mathrm{~h}^{\prime}\right) \mathrm{w}(\mathrm{w}) \mathrm{ig}$, seco y semicálido, con lluvias de verano, precipitación anual promedio de $800 \mathrm{~mm}$ y temperatura anual promedio de 18 ${ }^{\circ} \mathrm{C}$. La región presenta montañas y cerros con pendientes pronunciadas de hasta $60^{\circ}$, en altitudes que van de $560 \mathrm{~m}$ a $1580 \mathrm{~m} \mathrm{snm}$. El sustrato es sedimentario, superficial, localizado a menos de $15 \mathrm{~cm}$ de profundidad; dominan los suelos Leptosol y Rendzina (Instituto Nacional de Estadística y Geografía [Inegi] 2009). El área está conformada por parches de vegetación compuestos por campos agrícolas y bosques secundarios en las planicies próximas al Río Balsas, así como bosques primarios relativamente bien conservados en las zonas montañosas más alejadas. La vegetación dominante es bosque tropical caducifolio con predominio de las familias Fabaceae, Burseraceae y Poaceae, pero también hay Matorral Xerófilo tipificado por cactáceas columnares (Beltrán-Rodríguez et al., 2017b).

\section{Diseño de muestreo}

Para el muestreo de las poblaciones de $H$. latiflora se establecieron seis unidades de muestreo (UM): tres en zonas sometidas a descortezamiento comercial de $H$. latiflora y tres en zonas sin cosecha de corteza. Cada UM fue 
de $20 \mathrm{~m} \times 100 \mathrm{~m}$ (0.20 ha; 0.60 ha por zona), y se eligieron al azar mediante mapeos participativos con recolectores locales (Beltrán-Rodríguez et al., 2015; Beltrán-Rodríguez et al., 2017b).

En cada sitio se censaron todos los individuos de $H$. latiflora diferenciándolos por su etapa de desarrollo. Con base en estudios previos (Beltrán-Rodríguez et al., 2015; Beltrán-Rodríguez et al., 2017b), se categorizaron como plántulas a los individuos con una altura total $(\mathrm{AT}) \leq 10 \mathrm{~cm}$ y diámetro basal $(\mathrm{DB}) \leq 0.5 \mathrm{~cm}$; los juveniles fueron individuos no reproductivos y sin evidencias de haberse reproducido, con $\mathrm{AT} \geq 10.1 \mathrm{~cm} \leq 3 \mathrm{~m}$ y DB $\geq 0.6 \mathrm{~cm} \leq 8$ $\mathrm{cm}$; mientras que los adultos fueron plantas reproductivas con $\mathrm{AT} \geq 3.1 \mathrm{~m}$ y $\mathrm{DB} \geq 8.1 \mathrm{~cm}$. De cada individuo se registraron sus coordenadas (x,y) en un plano cartesiano, donde la esquina inferior izquierda del plano con respecto a la pendiente de cada UM representó el origen $(0,0)$.

\section{Análisis de datos}

Estructura poblacional. Todos los individuos previamente censados en las UM se organizaron en histogramas de frecuencias para representar la estructura poblacional en sitios cosechados y no cosechados. Previo a ello y con base en las recomendaciones de Beltrán-Rodríguez et al. (2018c), se calcularon puntos de inflexión en las curvas de acumulación del número de individuos de $H$. latiflora por DB en cada UM, con el objeto de subdividir naturalmente las diferentes etapas de desarrollo de H. latiflora y representar gráficamente, en forma más robusta, la distribución. Además, se calculó el sesgo y la curtosis como medidas descriptivas para determinar la influencia del aprovechamiento sobre la curva de distribución de cada población. La diferencia estadística de la estructura poblacional entre sitios por efecto de la cosecha se evaluó mediante una Prueba de $\chi^{2}$ con el Paquete R (R Core Team, 2019).

Distribución espacial. La distribución espacial de $H$. latiflora en sitios cosechados y no cosechados fue analizada con base en las coordenadas ( $\mathrm{x}, \mathrm{y}$ ) de cada individuo registrado por UM, utilizando la función $K(t)$ de Ripley
(Ripley, 1977). La función univariante $K(t)$ de Ripley utiliza un método de distancias entre todos los pares de árboles, tomando todas las distancias de los individuos registrados. Mediante esta función se obtiene información detallada, debido a la medición exacta de las coordenadas de todos los individuos presentes en cada UM, reflejando el tipo, escala e intensidad del patrón espacial que se presenta dentro de un círculo de radio $t$ (Ripley, 1977). De manera que la función $K(t)$ de Ripley representa el número de árboles que se encuentran en un círculo de radio variable $(t)$ alrededor de un determinado árbol (Ripley, 1981). $\lambda K(d)$ es la esperanza del número de individuos que están a una distancia igual o menor que $t$. La función empírica obtenida a partir de los datos reales de la UM se compara con la función obtenida en unidades simuladas mediante un proceso aleatorio; de esta forma se pueden determinar las tipologías de las estructuras que aparecen a diferentes escalas.

$$
\begin{gathered}
\lambda \cdot K(d)=\sum_{i=1}^{n} \sum_{j=1}^{n} \frac{\delta_{i j}(d)}{n}, i \neq j, \\
\delta_{i j}(d)\left[\begin{array}{l}
1 \text { si } d_{i j} \leq d \\
1 \text { si } d_{i j}>d
\end{array}\right]
\end{gathered}
$$

donde:

$\lambda=$ densidad o número de individuos de $H$. latiflora por hectárea

$d_{i j}=$ distancia entre el árbol $i$ y el $j$

$n=$ número de árboles del círculo de radio $t$

Ripley (1977) propuso una corrección del efecto del límite de la UM. Así, el valor resultante para cada $t$ se comparó con el valor de $K(t)$ esperado en una distribución de Poisson; por lo que este último y su intervalo de confianza se establecieron mediante 999 simulaciones tipo Monte Carlo (Besag y Diggle, 1977).

Besag (1977), a su vez, propuso utilizar la raíz cuadrada de la función $K(t)$ debido a que, en el supuesto de una distribución tipo Poisson, esta tendría un comportamiento 
lineal que facilitaría la comparación entre distintas distribuciones. La siguiente transformación tiene media $(\mu)$ cero y varianza $(\sigma)$ aproximadamente constante cuando la distribución es aleatoria:

$$
L(t)=\sqrt{\frac{K(t)}{\pi}-t}
$$

La comparación gráfica entre sitios cosechados y no cosechados de $H$. latiflora se representa en series de figuras, que describen la evolución de la función $L(t)$ con la distancia $(t)$ para una UM y los intervalos de confianza de la distribución tipo Poisson generados a partir de las simulaciones Monte Carlo (Besag y Diggle, 1977); donde: $K(t)=$ función de Ripley y $t=$ distancia entre dos individuos (m). Si el valor de $L(t)=0$ el patrón espacial con radio $t$ será aleatorio, valores de $L(t)>0$ indican agrupación y aquellos de $L(t)<0$ indican distribución uniforme. La hipótesis nula del análisis es la aleatoriedad espacial, de este modo si los valores obtenidos en $L(t)$ se encuentran fuera del límite de confianza se rechaza la hipótesis nula.

\section{Correlación espacial}

La relación espacial entre las distintas etapas de desarrollo (plántula, juvenil y adulto) de $H$. latiflora en sitios cosechados y no cosechados se analizó con la función bivariante $K_{12}(t)$ de Ripley (Lotwich y Silverman, 1982). Este índice se basa en el método de distancia al vecino más próximo. La hipótesis nula del análisis bivariado es la independencia espacial, indicando que un lugar dado es ambientalmente igual que otro para el establecimiento de las diferentes etapas de desarrollo (Greig-Smith, 1979; Lotwick y Silverman, 1982; Goreaud y Pélissier, 2003). La función $K_{12}(t)$ se define como:

$$
K_{12}(t)=\left(n_{1} n_{2}\right)^{-1}|A| \sum_{i=1}^{n_{1}} \sum_{j=1}^{n_{2}} W_{i j}^{-1} I_{t}\left(u_{i j}\right)
$$

Esta función índica atracción entre dos tipos de objetos si la distribución de puntos del tipo 1 ocurren cerca de los puntos o viceversa $\left(K_{12}(t)>\pi \mathrm{r}^{2}\right)$. Si ocurre lo contrario $\left(K_{12}(t)<\pi \mathbf{r}^{2}\right)$, entonces se puede afirmar repulsión entre los dos tipos. En caso de $K_{12}(t)=\pi \mathrm{r}^{2}$, ambos tipos de objetos son espacialmente independientes. Al igual que en la función univariante, la interpretación de esta función se facilita al linealizarla $\left(L_{12}(t)\right.$ ) (Szmyt, 2014; Velázquez, Martínez, Getzin, Moloney y Wiegand, 2016). La expresión es la siguiente:

$$
L_{12}(t)=\sqrt{\frac{K_{12}(t)}{\pi}-t}
$$

donde:

$K_{12}(t)=$ función de Ripley

$t=$ distancia

Un valor de $L_{12}(t)=0$ indica que los dos grupos son espacialmente independientes; $L_{12}(t)>0$ asociación positiva (atracción), y $L_{12}(t)<0$ asociación negativa (repulsión) (Besag y Diggle, 1977). Los patrones univariados y bivariados de Ripley se generaron con el programa Toolbox (Fisher, 2000).

\section{Resultados}

La densidad poblacional de H. latiflora considerando todas las etapas de desarrollo fue mayor en los sitios no cosechados $v s$. cosechados, $\left(\chi^{2}=8.58, \mathrm{gl}=2, \mathrm{p}<0.013\right)$; no obstante, el número de plantas juveniles e individuos reproductivos es menor (108vs. 152, respectivamente). La estructura poblacional entre sitios es semejante, con un patrón de distribución asimétrico a la derecha. Las estadísticas descriptivas de la curva de distribución indican que ambas poblaciones tienen asimetría positiva $\left(S^{3}=0.947\right.$ y $\left.S^{3}=1.063\right)$ y son leptocúrticas $\left(S^{4}=2.006\right.$ y $\left.S^{4}=2.400\right)$ (Fig. 1 a y b). 


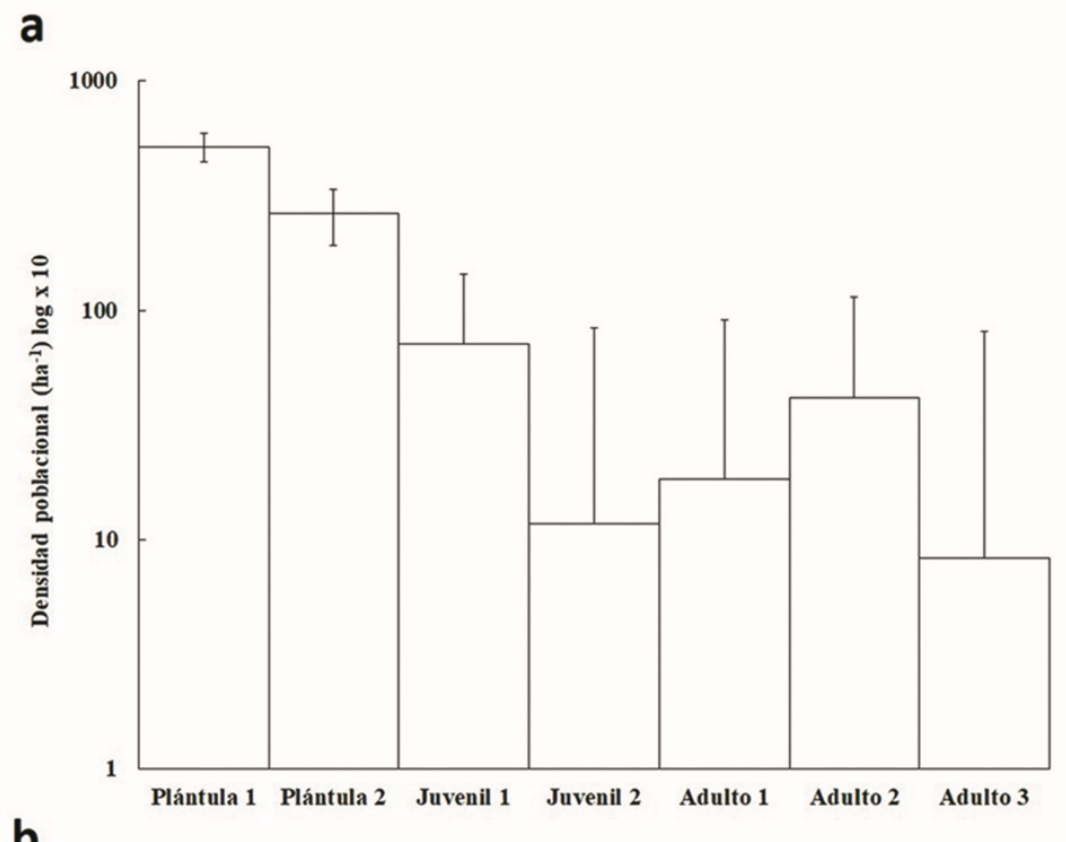

b

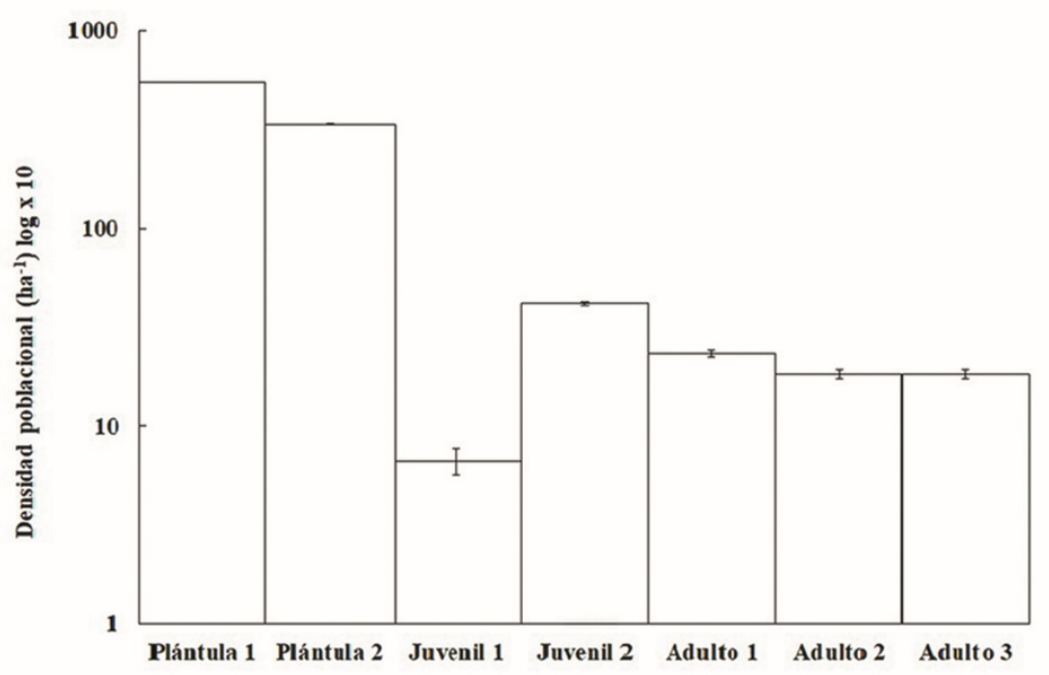

FIGURA 1. Estructura poblacional de Hintonia latiflora en sitios cosechados (a) vs. no cosechados (b). Número de individuos por hectárea \pm D.E. Intervalos de clase: Plántula $1=0.01 \mathrm{~cm}-0.15 \mathrm{~cm}$ de diámetro basal (DB); Plántula $2=0.16 \mathrm{~cm}-0.50 \mathrm{~cm}$ de DB; Juvenil $1=0.51 \mathrm{~cm}-4.85 \mathrm{~cm}$ de DB; Juvenil $2=4.86 \mathrm{~cm}-8.10 \mathrm{~cm}$ de DB; Adulto $1=$ $8.11 \mathrm{~cm}-13.40 \mathrm{~cm}$ de DB; Adulto $2=13.41 \mathrm{~cm}-21.0 \mathrm{~cm}$ de DB; Adulto $3=21.01 \mathrm{~cm}$ $-35.90 \mathrm{~cm}$ de DB.

El patrón de distribución univariante en todos los casos fue agregado $(L(t)>0)$, lo que indica que aparentemente la cosecha de corteza en esta especie no afecta la estructura espacial (Fig. 2 a-f). Sin embargo, la correlación espacial entre etapas de desarrollo de H. latiflora mostró un patrón diferente. En poblaciones sujetas a descortezamiento, el comportamiento refleja una combinación entre independencia y atracción espacial a diferentes distancias $(p$ $\leq 0.001)$, con marcada tendencia hacia la atracción de juveniles-adultos entre los $0.5 \mathrm{~m}$ y $2.5 \mathrm{~m}$ y los $4 \mathrm{~m}$ y $10 \mathrm{~m}$, 
así como de las plántulas-juveniles entre los $2.5 \mathrm{~m} \mathrm{y} 6 \mathrm{~m}$ y los 8 m y 10 m (Fig. 3 a-c y h-j). En cambio, en los sitios sin aprovechamiento de corteza se aprecia un evidente patrón de independencia espacial $\left(L_{12}(t)=0\right)$, salvo en el caso de una UM, en la que la interacción juveniles-adultos y plántulas-adultos refleja atracción espacial $(\mathrm{p} \leq 0.001)$ (Fig. 4 e y h).
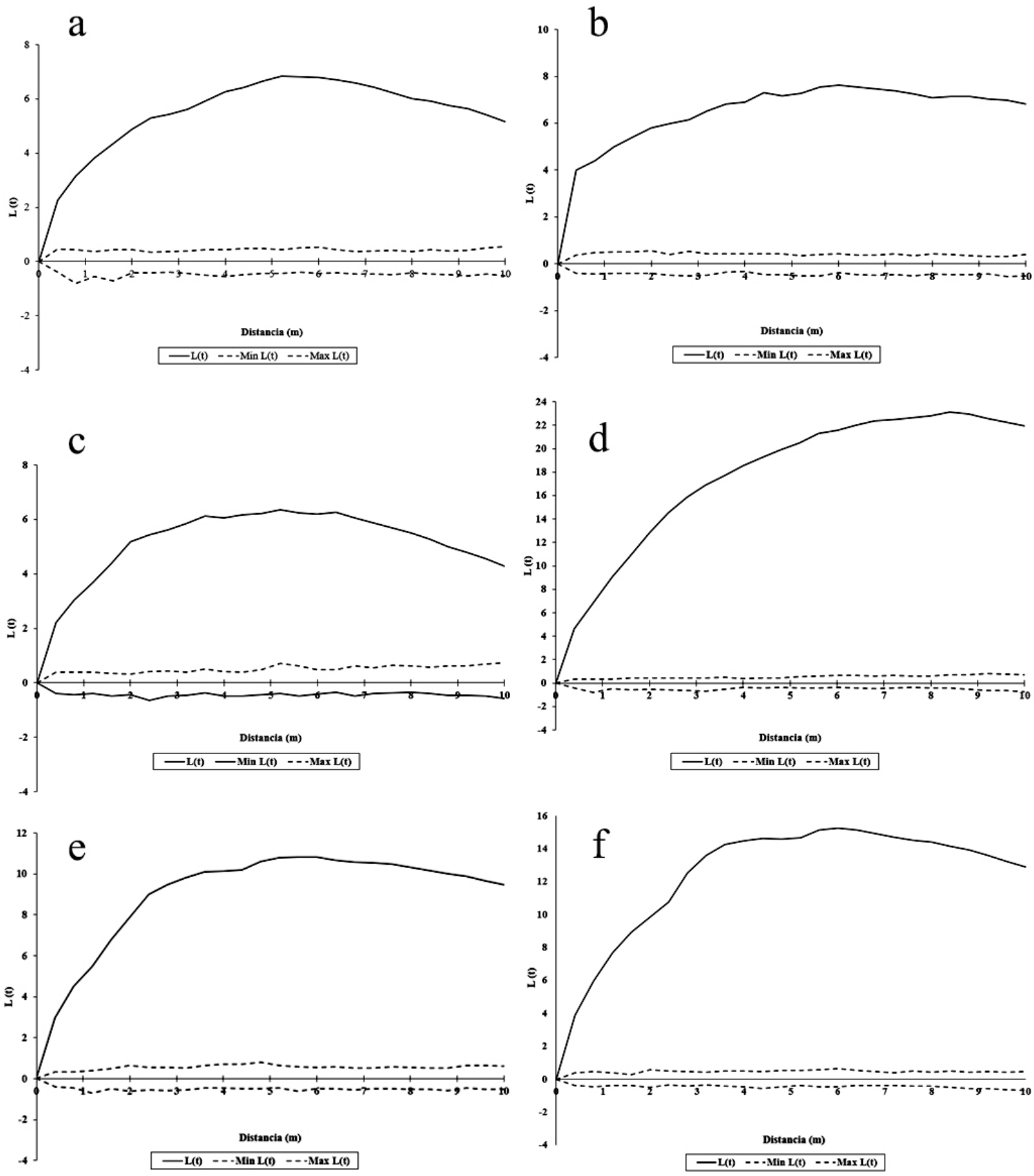

FIGURA 2. Patrón de distribución espacial univariante de Hintonia latiflora en sitios cosechados (a-c) vs. no cosechados (d-f). 

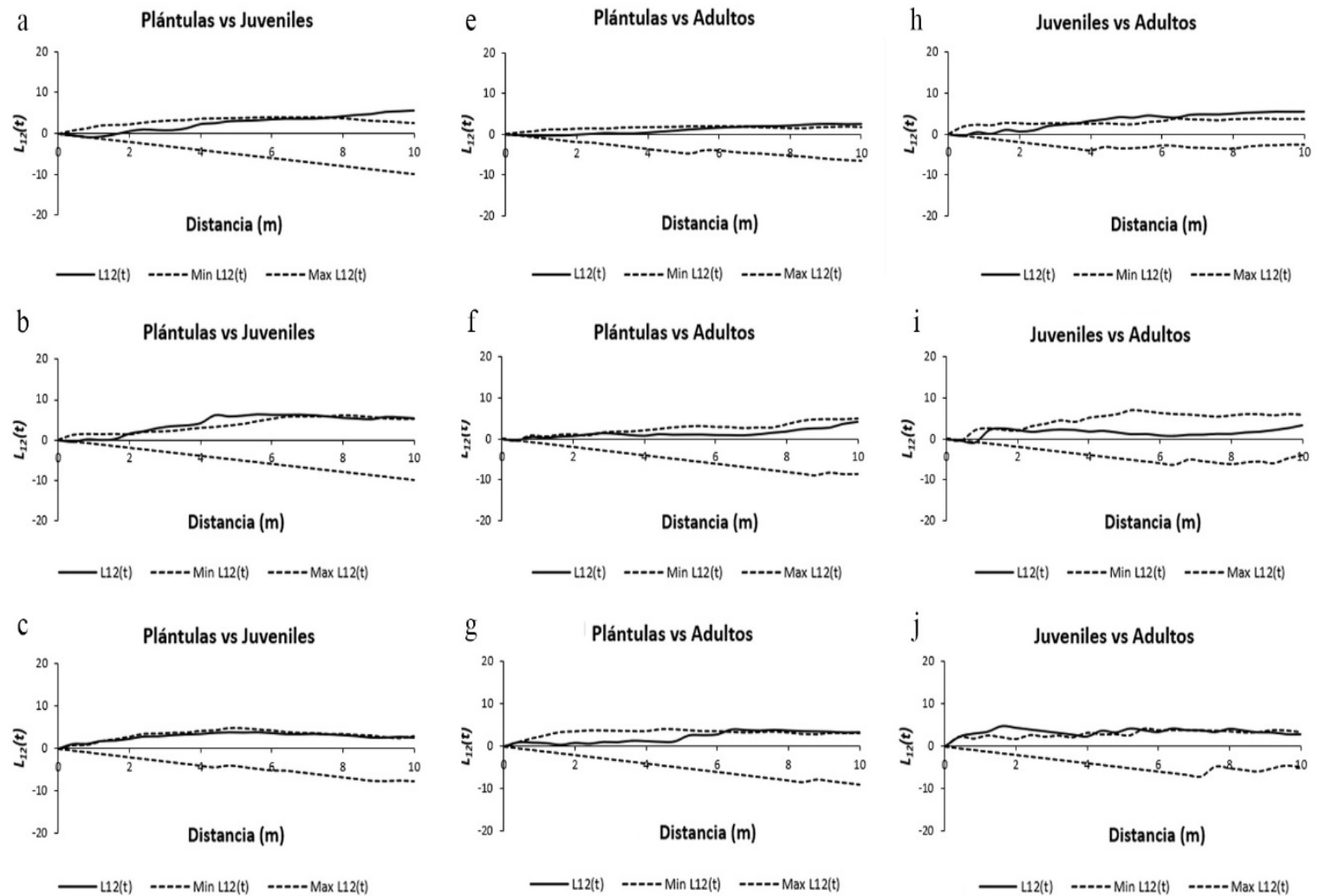

Figura 3. Patrón de correlación espacial $\left(L_{12}(t)\right)$ de Hintonia latiflora en sitios cosechados. Plántulas refiere a individuos con una altura total $(\mathrm{AT}) \leq 10 \mathrm{~cm}$ y diámetro basal $(\mathrm{DB}) \leq 0.5 \mathrm{~cm}$; juveniles son individuos no reproductivos con AT $\geq$ $10.1 \mathrm{~cm} \leq 3 \mathrm{~m} 2 \mathrm{y} \mathrm{DB} \geq 0.6 \mathrm{~cm} \leq 8 \mathrm{~cm}$; mientras que adultos son plantas reproductivas con $\mathrm{AT} \geq 3.1 \mathrm{~m}$ y DB $\geq 8.1 \mathrm{~cm}$

\section{DISCUSIÓN}

\section{Estructura poblacional en especies sujetas a aprovechamiento comercial}

Los resultados obtenidos de distribución diamétrica con asimetría a la derecha para los sitios cosechados y no cosechados de $H$. latiflora contrastan con la hipótesis planteada, ya que en ambas condiciones existe un alto número de individuos en las primeras categorías diamétricas y con disminución constante conforme se incrementa el tamaño (asimetría positiva). Este tipo de comportamiento coincide con lo encontrado por autores como Schumman, Wittig, Thiombiano, Becker y Hahn (2011) para la especie no maderable Anogeissus leiocarpa (DC.) Guill. \& Perr., quienes argumentan que el movimiento del suelo por actividades agrícolas favorece la incorporación de un mayor número de individuos en las categorías de menor tamaño, aun cuando estos son dañados y en ocasiones removidos en su totalidad del terreno.

Asimismo, Savi et al. (2018) registraron la misma distribución para Cola nitida (Vent.) Schott \& Endl. en el este de África, tomando en cuenta que la principal estructura utilizada en esta especie fueron las semillas y en menor intensidad el tallo y la corteza. Hallazgos similares son indicados por Botha, Witkowski y Shackleton (2004) y Gaoue y Ticktin (2007) para Catha edulis Forssk., Rapanea melanophloeos (L.) Mez y Khaya senegalensis (Desr.) A. Juss. en selvas tropicales de África. A diferencia de los resultados generados en la estructura poblacional de $H$. latiflora, Amahowe, Biaou, Natta y Balagueman (2017) encontraron para Afzzelia africana Sm. una distribución estadísticamente 
normal, con mayor número de individuos en las categorías intermedias en diferentes intensidades de disturbios.

En todas las investigaciones previamente mencionadas también se determinó que las densidades fueron más altas en las poblaciones cosechadas (Botha et al., 2004; Gaoue y Ticktin, 2007; Schumman et al., 2011; Amahowe et al., 2017; Savi et al., 2018;), lo que difiere con el presente estudio, debido a que se encontró un mayor número de individuos (particularmente plántulas) en sitios no cosechados. Esta situación podría deberse a los requerimientos específicos de cobertura vegetal y proporción de luz directa para la emergencia masiva y el desarrollo de las plántulas de $H$. latiflora (Beltrán-Rodríguez et al., 2015), condición que se presenta sobre todo en ambientes sin cosecha o poco intervenidos y bajo el dosel de la planta madre o de las especies con las que cohabita.

No obstante, en los sitios no cosechados se observó una baja cantidad de individuos juveniles y adultos, particularmente plantas multiramificadas con menor producción de corteza, posiblemente debido a las limitaciones biofísicas registradas en estos ambientes, tales como pendientes abruptas, suelos superficiales y altamente pedregosos (Beltrán-Rodríguez et al., 2017), las cuales impactan en las tasas de supervivencia de las plántulas y en su eventual crecimiento, como ha sido evaluado para diversas especies de los bosques tropicales caducifolios (Gonçalves, Revermann, Cachissapa, Gomes y Aidar, 2018).
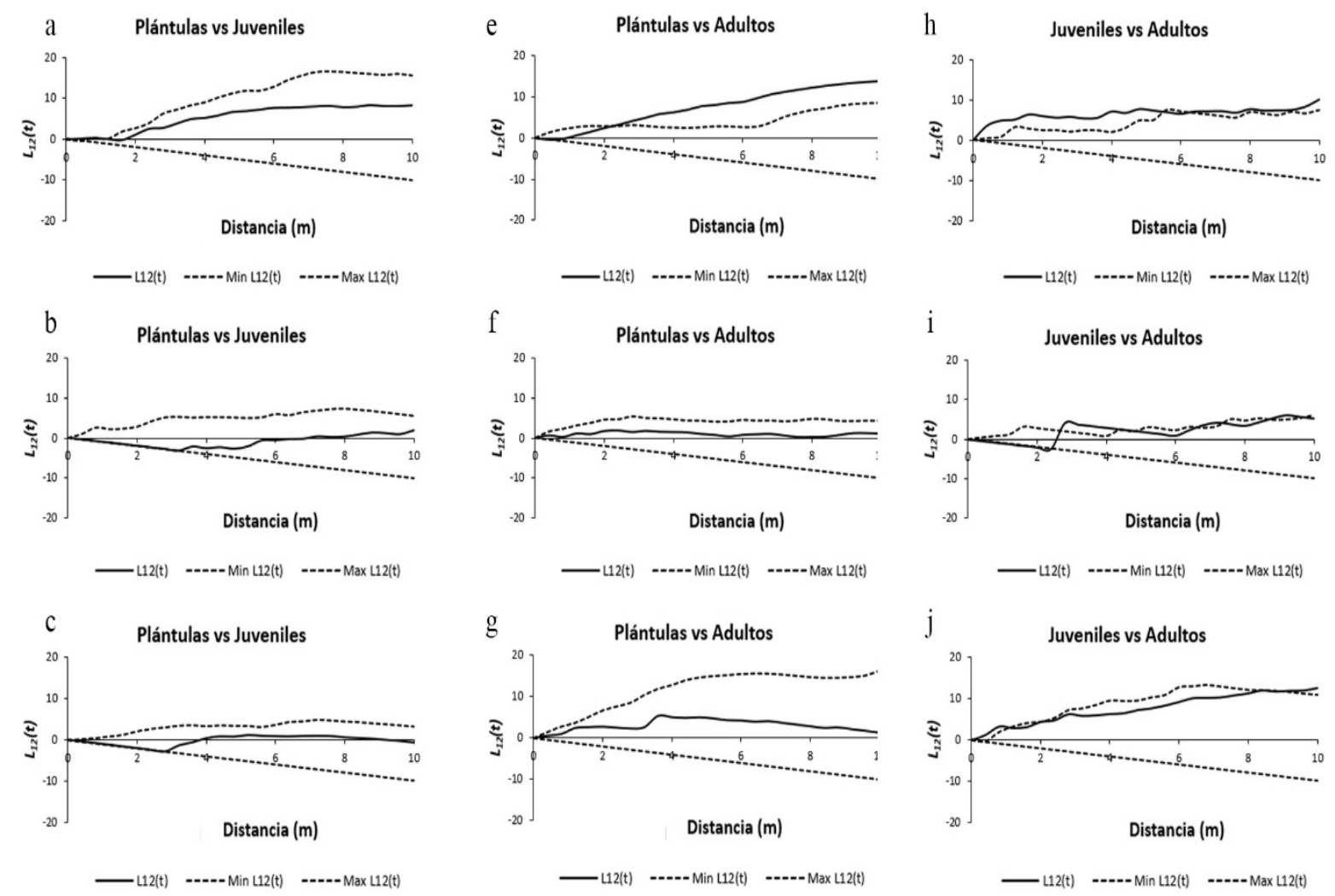

FIGURA 4. Patrón de correlación espacial $\left(L_{12}(t)\right)$ de Hintonia latiflora en sitios no cosechados. Plántulas refiere a individuos con una altura total $(\mathrm{AT}) \leq 10 \mathrm{~cm}$ y diámetro basal $(\mathrm{DB}) \leq 0.5 \mathrm{~cm}$; juveniles son individuos no reproductivos con AT $\geq 10.1 \mathrm{~cm} \leq 3 \mathrm{~m}$ y DB $\geq 0.6 \mathrm{~cm}$ $\leq 8 \mathrm{~cm}$; mientras que adultos son plantas reproductivas con $\mathrm{AT} \geq 3.1 \mathrm{~m}$ y DB $\geq 8.1 \mathrm{~cm}$. 
Por el contrario, en los sitios cosechados la densidad de juveniles y adultos de H. latiflora fue mayor, quizá debido a la dominancia de plantas con porte monopódico presentes en áreas de poco acceso, así como a la presencia de individuos polifurcados reiterados por el aprovechamiento (Beltrán-Rodríguez et al., 2017). De manera que, como lo sugieren Beltrán-Rodríguez et al. (2017b), el descortezamiento sostenible en esta especie estimula la reiteración, y, en consecuencia, permite el mantenimiento de las poblaciones.

En concordancia con estos resultados, Gueje, Tchamou y Lejoly (2016) encontraron en Camerún que para Garcinia lucida Vesque, el uso de machete para el desprendimiento de la corteza favorece la sobrevivencia y recuperación de las heridas, debido a que solo se afecta la corteza externa y no daña el cambium vascular, contrario a si el aprovechamiento se realiza en forma destructiva, lo cual impacta negativamente en la regeneración (Lynser y Tiwari, 2016; Dao y Hölscher, 2018). Con base en lo expuesto, es posible plantear que el descortezado hasta ahora no ha limitado el buen potencial de reproducción y la repoblación natural en la mayoría de las especies arbóreas tropicales estudiadas.

\section{Efectos de la cosecha sobre la distribución y correlación espacial de Hintonia latiflora}

Contrario a la hipótesis planteada, el patrón de distribución espacial de $H$. latiflora no se vio afectado por el aprovechamiento de corteza, ya que en ambos casos fue agregado. Esto se explica por el mecanismo de diseminación dominante que presenta esta especie (barocoria), el cual, de acuerdo con Beltrán-Rodríguez et al. (2015), propicia germinación masiva bajo la planta madre y, en consecuencia, favorece la agregación espacial. Este resultado es similar a lo demostrado por Peres y Baider (1997) para Bertholletia excelsa Bonpl. en Brasil, dado que en este estudio la distancia máxima promedio de dispersión fue menor o igual a $5 \mathrm{~m}$. También coincide con lo registrado por Réjou-Méchain et al. (2011) para 106 especies arbóreas tropicales de Camerún y África Central, cuya agregación espacial está asociada estrechamente al síndrome de dispersión, particularmente dispersión limitada (Fibich et al., 2016).

La agregación espacial de $H$. latiflora también puede ser explicada por la capacidad de reiteración que presentan los individuos en etapa adulta una vez cosechados, ya que de los tocones se regeneran árboles polifurcados (BeltránRodríguez et al., 2017b); mismos que mantienen su posición inicial en el espacio. Roland, Loh, Enow, Bechem y Yengo (2013) demostraron que para nueve especies arbóreas tropicales utilizadas como RFNM, la agregación espacial es más evidente cuando las especies son poco abundantes, pero con una alta capacidad de regeneración, como en el caso de $H$. latiflora, que alcanza una densidad de apenas 5 ind. ha ${ }^{-1}$ en etapa reproductiva (Beltrán-Rodríguez et al., 2017b), aun cuando su densidad en renuevos vía semilla y multirramificación supera la densidad de juveniles y adultos en el sitio, como ha sido observado en este estudio.

De igual forma, Elias, Dias y Pereira (2011) encontraron que para las especies arbóreas que se distribuyen en las montañas de los Azores, los regímenes de disturbio del bosque y las estrategias de regeneración de cada especie explican su patrón de distribución espacial, siendo las especies pioneras y heliófitas, como $H$. latiflora (Beltrán-Rodríguez et al., 2015), más agregadas debido a la dependencia de claros para emerger e incorporar nuevos individuos a la población.

A pesar de lo anterior, es importante precisar, como lo sugieren Réjou-Méchain et al. (2011), que la escala espacial de análisis es un factor relevante que también puede influir en el patrón de distribución de una especie, ya que, como se demostró para otro grupo de RFNM del género Copaifera spp. en la Amazonía Brasileña, dicho patrón puede variar a nivel intra e interespecífico si se analiza a escala local o a nivel de paisaje. Particularmente para el caso de especies bajo aprovechamiento comercial, integrar también al análisis el papel que juega la dinámica en el uso de la tierra como estrategia de estructuración de los paisajes culturales podría aportar conocimiento sobre su distribución espacial, como lo proponen Dalle, López, Díaz, Legendre y Potvin 
(2002) para 23 especies útiles que utilizan los indígenas Kuna en Panamá.

En consecuencia, futuros estudios deben enfocarse a comprender la dinámica espacial de H. latiflora en México y, con base en ello, generar hipótesis sobre los mecanismos socioecológicos que la determinan. Esta información tendría implicaciones importantes para los recolectores de H. latiflora, dado que, de mantenerse en un patrón agrupado en etapa adulta, independientemente de la escala de análisis, se esperarían abundantes cosechas de corteza en menor distancia, incrementando así la eficiencia y viabilidad económica del proceso de aprovechamiento. Esta conclusión es parcial y momentánea, mientras se demuestran los efectos de la densodependencia conforme los individuos crecen a la etapa adulta.

Finalmente, con respecto a la distribución bivariante de $H$. latiflora en sitios con diferente grado de manejo (cosechados vs. no cosechados), también se rechaza la hipótesis planteada, debido a que en las poblaciones bajo descortezamiento comercial se registró atracción espacial a diferentes distancias y entre distintas etapas de desarrollo. Particularmente en estas áreas, los resultados aquí obtenidos son similares a los registrados por Fuchs, Robles y Hamrick (2013) para la especie medicinal Guaiacum sanctum L. en Costa Rica, debido a que las plántulas fueron encontradas predominantemente bajo el dosel del árbol progenitor. También coinciden con lo observado por Cheng, Han, Kang, Song y Liu (2014) en China, ya que estos autores encontraron asociaciones positivas a pequeña escala entre plántulas-juveniles y juveniles-adultos de Quercus liaotungensis Koidz.

Como lo sugiere Beltrán-Rodríguez et al. (2015), la sombra proporcionada por la planta madre u otros individuos conespecíficos de menor talla facilita el establecimiento de H. latiflora; con el paso del tiempo y el crecimiento de las plantas, estos individuos tienden hacia la independencia espacial (probable densodependencia) para evitar competencia por recursos lumínicos e hídricos, como ocurre con las poblaciones no cosechadas. Pero, en ambientes manejados, algunos individuos juveniles mantendrán una estrecha interacción espacial con los adultos, debido a que el descortezamiento se lleva a cabo en ambas etapas de desarrollo (Beltrán-Rodríguez et al., 2017b). Lo anterior propicia un cambio en la dirección natural de la estructura espacial de este RFNM, promoviendo una dependencia positiva (atracción), posiblemente para tolerar condiciones de estrés fisiológico derivadas por la cosecha y por las condiciones ambientales propias de sitios intervenidos (Quesada et al., 2011).

\section{CONCLUSIONES}

El descortezamiento de $H$. latiflora no tiene efecto sobre la estructura poblacional ni sobre el patrón de distribución espacial univariante, pero sí sobre la correlación espacial entre etapas de desarrollo, con particular impacto en los sitios cosechados, en los que se registra mayor densidad de individuos juveniles y adultos, pero menor incorporación de plántulas, así como una interacción espacial positiva (atracción) entre plántulasjuveniles y juveniles-adultos. Esta contribución representa uno de los primeros esfuerzos a escala nacional para comprender el impacto de la cosecha de especies medicinales comercializadas como RFNM en su estructura poblacional-espacial, y proporciona información que puede ser utilizada para el manejo sostenible de H. latiflora en áreas sujetas a descortezamiento comercial.

\section{RECONOCIMIENTOS}

Esta investigación fue financiada parcialmente por el Consejo Nacional de Ciencia y Tecnología de México (becario Conacyt 265032) y por el Colegio de Postgraduados Campus Montecillo. Se agradece también a la Dirección General de Asuntos del Personal Académico (DGAPA) de la UNAM por la beca posdoctoral otorgada al autor de correspondencia, misma que permitió trabajar en la redacción del presente documento. Finalmente se expresa un profundo agradecimiento a las autoridades comunales de Tlalcozotitlán, así como a Emiliano Soriano y Roberto Flores por su apoyo en el trabajo de campo. 


\section{REFERENCIAS}

Arellanes-Cancino, Y., Romero-Sosa, M. A., Vega, E., Maza-Villalobos, S., \& Casas-Fernández, A. (2018). Ecological bases for sustainable management of Pochote (Ceiba aesculifolia subsp. parvifolia) through demographic analysis. Economic Botany, 72(1), 20-37.

Amahowe, O. I., Biaou, S. S., Natta, A. K., \& Balagueman, R. O. (2017). Multiple disturbance patterns and population structure of a tropical tree species, Afzelia africana (LeguminosaeCaesalpinioideae), in two contrasting bioclimatic zones of the Republic of Benin. Southern Forests: a Journal of Forest Science, 80(2), 95-103. doi: 10.2989/20702620.2016.1274940

Badii, M. H., Guillen, A., Cerna, E., \& Landeros, J. (2011). Dispersión Espacial: El Prerrequisito Esencial para el Muestreo. Daena: International Journal of Good Conscience, 6(1), 40-71.

Beltrán-Rodríguez, L., Romero-Manzanares, A., Luna-Cavazos, M., Vibrans, H., Manzo-Ramos, F., Cuevas-Sánchez, J., \& GarcíaMoya, E. (2015). Historia Natural y Cosecha de Corteza de Quina Amarilla Hintonia latiflora (Rubiaceae). Botanical Sciences, 93(2), 261 272. doi: 10.17129 /botsci.231

Beltrán-Rodríguez, L., Manzo-Ramos, F., Maldonado-Almanza, B., Martínez-Ballesté, A., \& Blancas, J. (2017a). Wild Medicinal Species Traded in the Balsas Basin, Mexico: Risk Analysis and Recommendations for Their Conservation. Journal of Ethnobiology, 37(4), 743-764. doi: 10.2993/0278-0771-37.4.743

Beltrán-Rodríguez, L., Romero-Manzanares, A., Luna-Cavazos, M., \& García-Moya, E. (2017b). Variación arquitectónica y morfológica de Hintonia latiflora (Rubiaceae) en relación a la cosecha de corteza y factores ambientales. Revista de Biología Tropical 65(3), 900-916. doi: $10.15517 /$ rbt.v65i3.29444

Beltrán-Rodríguez, L. (2018a). Estructura, dinámica poblacional y regeneración del leño de Amphipterygium adstringens (Anacardiaceae) en el ejido El Limón, Morelos, México. Tesis de doctorado, Colegio de Postgraduados Campus Montecillo, Texcoco de Mora, Estado de México.

Beltrán-Rodríguez, L., Maldonado-Almanza, B., Cristians, S., Blancas, J., Sierra-Huelz, A., \& Bye, R. (2018b). Las cortezas como productos forestales no maderables en México: Análisis nacional y recomendaciones para su aprovechamiento sostenible. D.F., México: Consejo Nacional de Ciencia y Tecnología (Conacyt No. 293914).

Beltrán-Rodríguez, L., Valdez-Hernández, J., Luna-Cavazos, M., Romero-Manzanares, A., Pineda-Herrera, E., MaldonadoAlmanza, B., Borja de la Rosa, Ma., \& Blancas-Vázquez, J. (2018c). Estructura y diversidad arbórea de bosques tropicales caducifolios secundarios en la Reserva de la Biósfera Sierra de Huautla, Morelos. Revista Mexicana de Biodiversidad, 89(1), 108-122. doi: 10.22201/ib.20078706e.2018.1.2004
Besag, J. (1977). Contribution to the discussion on Dr. Ripley's paper. Journal of the Royal Statistical Society, B 39, 193-195.

Besag, J. \& Diggle, P. J. (1977). Simple Monte Carlo tests for spatial pattern. Applied Statistics 26, 327-333.

Botha, J., Witkowski, E., \& Shackleton, C. (2014). Harvesting impacts on commonly used medicinal tree species (Catha edulis and Rapanea melanophloeos) under different land management regimes in the Mpumalanga Lowveld, South Africa. Koedoe, 47, 1-18.

Blancas, J., Caballero, J., \& Beltrán-Rodríguez, L. (2017). Los productos forestales no maderables de México. Panorama General. Red Temática Productos Forestales No Maderables. D.F., México: Consejo Nacional de Ciencia y Tecnología (Conacyt No. 280901).

Cheng, X., Han, H., Kang, F., Song, Y., \& Liu, K. (2014). Point pattern analysis of different life stages of Quercus liaotungensis in Lingkong Mountain, Shanxi Province, China. Journal of Plant Interactions, 9(1), 233-240. doi: 10.1080/17429145.2013.818167

Dao, T. H. H. \& Hölscher, D. (2018). Impact of Non-Timber Forest Product Use on the Tree Community in North-Western Vietnam. Forests, 9(431), 1-15. doi: 10.3390/f9070431

Dalle, S., López, H., Díaz, D., Legendre, P., \& Potvin, C. (2002). Spatial distribution and habitats of useful plants: an initial assessment for conservation on an indigenous territory, Panama. Biodiversity and Conservation, 11(4), 637-667. doi: 10.1023/A:101554432

Elias, R. B., Dias, E., \& Pereira, F. (2011). Disturbance, regeneration and the spatial pattern of tree species in Azorean mountain forests. Community Ecology, 12(1), 23-30. doi: 10.1556/ComEc.12.2011.1.4

Fibich, P., Lepš, J., Novotný, V., Klimeš, P., Têšitel, J., Molem, K., Damas, K., \& Weiblen, G. (2016). Spatial patterns of tree species distribution in New Guinea primary and secondary lowland rain forest. Journal of Vegetation Science, 27, 328-339. doi: $10.1111 /$ jvs. 12363

Fisher, M. 2000. Toolbox: Software available for sophisticated spatial statistics. University of the South Pacific. Suva, Fiji.

Fuchs, E., Robles, T., \& Hamrick, J. (2013). Spatial distribution of Guaiacum sanctum (Zygophyllaceae) seedlings and saplings relative to canopy cover in Palo Verde National Park, Costa Rica. International Journal of Tropical Biology, 61(3), 1521-1533. doi: 10.15517/RBT.V61I3.11981

Gaoue, O. G., \& Ticktin, T. (2007). Patterns of harvesting foliage and bark from the multipurpose tree Khaya senegalensis in Benin: Variation across ecological regions and its impacts on population structure. Biological Conservation, 137(3), 424-436. doi: 10.1016/j.biocon.2007.02.020 
García, E. 2004. Modificaciones al Sistema de Clasificación Climática de Köppen (para adaptarlo a las condiciones de la República Mexicana). Serie Libros. Núm. 6. Instituto de Geografía. D.F., México: Universidad Nacional Autónoma de México.

Gonçalves, F. M. P., Revermann, R., Cachissapa, M. J., Gomes, A. L., \& Aidar, M. P. M. (2018). Species diversity, population structure and regeneration of woody species in fallows and mature stands of tropical woodlands of southeast Angola. Journal of Forestry Research 29(6), 1569-1579. doi: 10.1007/s11676-018-0593-x

Goreaud, F. \& Pélissier, R. (2003). Avoiding misinterpretation of biotic interactions with the intertype $K_{12}$-function: population independence vs. random labelling hypothesis. Journal of V egetation Science, 14, 681-692. doi: 10.1111/j.1654-1103.2003.tb02200.x

Guedje, N. M., Tchamou, N., \& Lejoly, J. (2016). Tree response to bark harvest: the case of a medicinal species, Garcinia lucida, as source of raw materials for plant-based drug development. Journal of Applied Biosciences, 99, 9476-9491. doi: 10.4314/jab.v99i1.13

Greig-Smith, P. (1979). Pattern in vegetation. Journal of Ecology, 67, 755779 .

Hernández-Apolinar, M., Valverde, T., \& Purata, S. (2006). Demography of Bursera glabrifolia, a tropical tree used for folk woodcrafting in Southern Mexico: An evaluation of its management plan. Forest Ecology and Management, 223(1-3), 139-151. doi: 10.1016/j.foreco.2005.10.072

Hersch-Martínez, P., \& Fierro, A. (2001). El comercio de plantas medicinales: algunos rasgos significativos en el centro de México. En B. Rendón, S. Rebollar, J. Caballero, \& M. Martínez-Alfaro (Eds.), Plantas, cultura y sociedad. Estudio sobre la relación entre los seres bumanos y las plantas de los albores del siglo XXI (pp. 53-75). D.F., México: UAM, Semarnat.

Instituto Nacional de Estadística y Geografía [Inegi]. (2009). Prontuario de información geográfica municipal de los Estados Unidos Mexicanos. Copalillo, Guerrero, México. Recuperado de http://www3.inegi.org.mx/contenidos/app/mexicocifras/datos geograficos/12/12019.pdf

Jansen, M., Anten, N. P. R., Bongers, F., Martínez-Ramos, M., \& Zuidema, P. A. (2018). Towards smarter harvesting from natural palm populations by sparing the individuals that contribute most to population growth or productivity. Journal of Applied Ecology, 55(4), 1682-1691. doi: 10.1111/1365-2664.13100

Koleff, P., Urquiza-Haas, T., \& Contreras, B. (2012). Prioridades de conservación de los bosques tropicales en México: reflexiones sobre su estado de conservación y manejo. Ecosistemas, 21(1-2), 620.
Lawes, M. J., Griffiths M. E., Midgley J. J., Boudreau S., Eeley H. A. C., \& Chapman, C. A. (2008). Tree spacing and area of competitive influence do not scale with tree size in an African rain forest. Journal of Vegetation Science, 19(5), 729-738. doi: 10.3170/2008-818445

Ledo, A., Condés, S., \& Montes, F. (2012). Revisión de índices de distribución espacial usados en inventarios forestales y su aplicación en bosques tropicales. Revista Peruana de Biología, 19(1), 113-124.

Lynser M. B., \& Tiwari B. K. (2016). Diversity and Utilization of Floral Non Timber Forest Products by the Communities in Rural Meghalaya, North-East India. Journal of Forest and Environmental Science, 32(1), 39-54. doi: 10.7747/JFES.2016.32.1.39

Lotwich, H. W., \& Silverman, B. W. (1982). Methods for analysing spatial processes of several types of points. Journal of the Royal Statistical Society, Series B: Methodological, 44(3), 406-413. doi: 10.1111/j.25176161.1982.tb01221.x

Lorence, D. H., \& Taylor, C. M. (2011). Rubiaceae. Flora Mesoamericana, 4, 230-232.

Mata, R., Navarrete, A., Cristians, S., Hersch, P., \& Bye, R. (2009). Plantas Medicinales de México. Monografía Cientifica. Pruebas de Control de Calidad (Identificación y Composición), Eficacia y Seguridad. Copalcbi Hintonia latiflora (Sessé et Mociño ex DC.) Bullock (Rubiaceae). D.F., México: Sentido Giratorio Ediciones.

Martínez-Ballesté, A., \& Martorell, C. (2015). Effects of Harvest on the Sustainability and Leaf Productivity of Populations of Two Palm Species in Maya Homegardens. PLOS ONE, 10(3), 1-17. doi: 10.1371/journal.pone.0120666

McIntire, E. J. B., \& Fajardo, A. (2009). Beyond description: the active and effective way to infer processes from spatial patterns. Ecology, 90(1), 46-56. doi: 10.1890/07-2096.1

Pavón-Hernández, N. P., \& Rico-Gray, V. (2004). Distribución espacial de Tilia mexicana Schlecht. (Tiliaceae) en el paisaje del centro del estado de Veracruz, México. Universidad y Ciencia, 20(39), 29-32.

Peres, C. \& Baider, C. (1997). Seed dispersal, spatial distribution and population structure of Brazilnut trees (Bertholletia excelsa) in southeastern Amazonia. Journal of Tropical Ecology, 13, 595-61. doi: 10.1017/S0266467400010749

Picard, N., Bar-Hen, A., Mortier, F., \& Chadoeuf, J. (2009). Understanding the dynamics of an undisturbed tropical rain forest from the spatial pattern of trees. Journal of Ecology, 97(1), 97-108. doi: 10.1111/j.1365-2745.2008.01445.x 
Quesada, M., Rosas, F., Aguilar, R., Ashworth, L., Rosas-Guerrero, V. M., Sayago, R., Lobo, J. A., Herrerías-Diego, Y., \& SánchezMontoya, G. (2011). Human Impacts on Pollination, Reproduction, and Breeding Systems in Tropical Forest Plants. En R. Dirzo, H. S. Young, H. A. Mooney \& G. Ceballos (Eds.), Seasonally Dry Tropical Forests. Ecology and Conservation (pp. 173-194). Washington DC, Estados Unidos: Island Press.

R Core Team. (2019). R: A language and environment for statistical computing. $\mathrm{R}$ Foundation for Statistical Computing, Vienna, Austria. Recuperado de https://www.r-project.org/

Rayburn, A. P., Schiffers, K., \& Schupp, E. W. (2011). Use of precise spatial data for describing spatial patterns and plant interactions in a diverse Great Basin shrub community. Plant Ecology, 212, 585594. doi: 10.1007/s11258-010-9848-0

Réjou-Méchain, M., Flores, O., Bourland, N., Doucet, J. L., Fétéke, R., Pasquier, A., \& Hardy, O. J. (2011). Spatial aggregation of tropical trees at multiple spatial scales. Journal of Ecology, 99, 1373-1381. doi: 10.1111/j.1365-2745.2011.01873.x

Ripley, B. D. (1977). Modeling spatial patterns (with discussion). Journal of the Royal Statistical Society, Series B: Methodological 39, 172-212.

Ripley, B. D. (1981). Spatial Statistics. New York, Estados Unidos: Wiley and Sons.

Roland, N., Loh, E., Enow, E., Bechem, E., \& Yengo, T. (2013). Spatial distribution and abundance of selected exploited non-timber forest products in the Takamanda National Park, Cameroon. International Journal of Biodiversity and Conservation, 5(6), 378-388. doi: 10.5897/IJBC2012.0540.

Savi, M. K., Noumonvi, R., Chadaré, F. J., Daïnou, K., Salako, V. K., Idohou, R., Assogbadjo A. E., Kakaï, R. G. (2018). Synergy between traditional knowledge of use and tree population structure for sustainability of Cola nitida (Vent.) Schott. \& Endl in Benin (West Africa). Environment, Development and Sustainability. doi: 10.1007/s10668-018-0091-5

Schumann, K., Wittig, R., Thiombiano, A., Becker, U., \& Hahn, K. (2011). Impact of land-use type and harvesting on population structure of a non-timber forest product-providing tree in a semi- arid savanna, West Africa. Biological Conservation, 144(9), 23692376. doi: 10.1016/j.biocon.2011.06.018

Szmyt, J. (2014). Spatial statistics in ecological analysis: from indices to functions. Silva Fennica, 48(1), 1-31. doi: 10.14214/sf.1008

Soberón, J., \& Miller, C. P. (2009) Evolución de los nichos ecológicos. Miscelánea Matemática, 49, 83-99.

Velázquez, E., Martínez, I., Getzin, S., Moloney, K. A., \& Wiegand, T. (2016). An evaluation of the state of spatial point pattern analysis in ecology. Ecography, 39(11), 1-14. doi: 10.1111/ecog.01579

van Lent, J., Hernández-Barrios, J. C., Anten, N. P. R., \& MartínezRamos, M. (2014). Defoliation effects on seed dispersal and seedling recruitment in a tropical rain forest understorey palm. Journal of Ecology, 102(3), 709-720. doi: 10.1111/1365-2745.12216

Zakaria, M., Rajpar, M. N., Ozdemir, I., \& Rosli, Z. (2016). Fauna Diversity in Tropical Rainforest: Threats from Land-Use Change. En J. A. Blanco, S. C. Chang \& Y. H. Lo (Eds.), Tropical forests the challenges of maintaining ecosystem services while managing the landscape (pp. 11-49). Croatia: InTech.

Manuscrito recibido el 6 de septiembre de 2019

Aceptado el 17 de enero de 2020

Publicado el 10 de junio de 2020

Este documento se debe citar como:

Vásquez-Cortez, V. F., Beltrán-Rodríguez, L., Ángeles-Pérez, G. Romero-Manzanares, A., Garcia-Moya, E., Luna-Cavazos, M., Caballero, J., Blancas, J., Martínez-Ballesté, A., \& MontoyaReyes, F. (2020). ¿El descortezamiento de un árbol medicinal impacta en su estructura poblacional-espacial? El caso de Hintonia latiflora en México. Madera y Bosques, 26(2), e2622037. doi: 10.21829/myb.2020.2622037

Madera y Bosques por Instituto de Ecología, A.C. se distribuye bajo una Licencia Creative Commons Atribución-NoComercialCompartirlgual 4.0 Internacional. 